\title{
EFFECT OF YOGA ON CORTICAL INHIBITION IN PATIENTS WITH DEPRESSION : A TRANS-CRANIAL MAGNETIC STIMULATION STUDY
}

\author{
JitenderJakhar, Urvakhsh Meherwan Mehta, BNGangadhar \\ Department of Psychiatry, NIMHANS, Bengaluru
}

Background and aims: Neurobiological underpinnings of depression are poorly understood .Dysregulation in cortical excitation/inhibition balance has been thought to be one of the putative markers of depression. Yoga therapy has been found to be effective in reducing symptoms of depression, but the mechanisms through which the therapeutic effects are mediated are currently poorly understood .Transcranial Magnetic Stimulation (TMS) provides a unique opportunity to noninvasively determine cortical inhibitory and excitatory interneuron functions in humans in a cost-effective manner. In this study, First we aim to examine TMS single- and paired-pulse derived cortical inhibitory functions in patients $(n=56)$ with moderate to severe unipolar depression (ICD-10) and a group of healthy comparison subjects $(n=34)$. Then compare TMS changes in depression patients, after one single session of yoga $(n=20)$ vs intermittent walking $(n=20)$.

Materials and methods:After determining the resting motor threshold,twenty recordings were obtained in a random sequence for each of the following cortical inhibition/excitation parameters: test pulse derived motor evoked potential (MEP), short interval intra-cortical inhibition (SICI), long interval intracortical inhibition (LICI), intra-cortical facilitation (ICF) and cortical silent period(CSP).Patients were also rated on severity of their depressive symptoms using the Hamilton Depression Rating Scale(HDRS)
Results: The two groups were comparable in terms of sex, resting motor threshold, $\mathrm{LICl}$ and ICF.However there were significant differences in CSP.Depression patients have shown significantly reduced CSP, as compared to healthy subjects with $\mathrm{p}<0.01$. SICl also has shown trend towards significance with lower value in patients with depression as compared to healthy subjects. Among depression patients, single session of yoga resulted in significant enhancement of CSP $(p<0.05)$, when compared to single session of intermittent walking.

Conclusions: Patients with depression have deficits in both $\mathrm{GABA}_{A}(\mathrm{SICl})$ and $\mathrm{GABA}_{B}(\mathrm{CSP})$ mediated neurotransmission as measured using TMS and this CSP deficit improves after single session of yoga, demonstrating that putative target engagement of yoga therapy by its action on GABAergic neurotransmitter system. This study also highlights for the first time an acute effect of yoga therapy in engagement of biological systems (GABA interneuron functions) using Transcranial Magnetic Stimulation

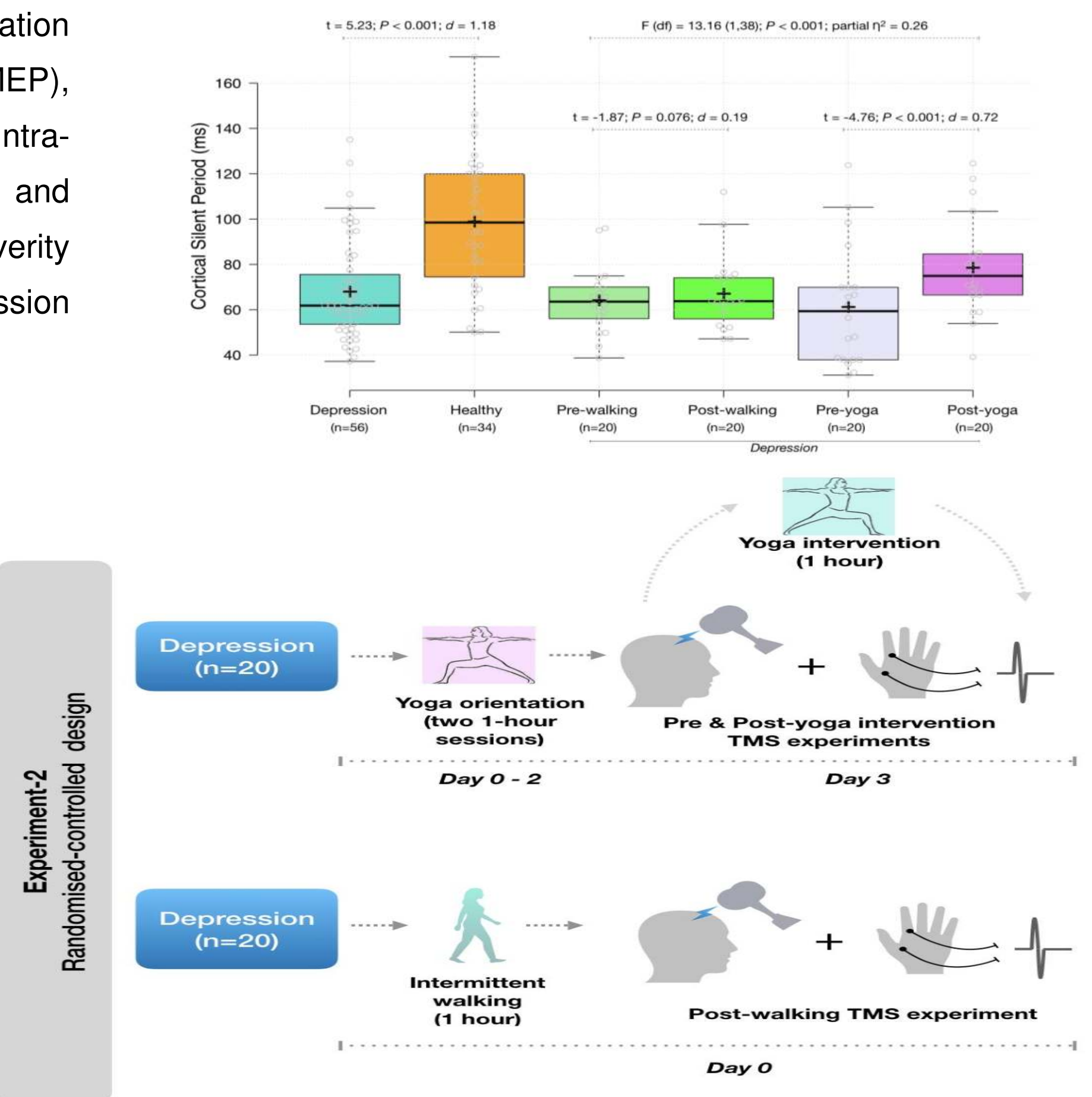

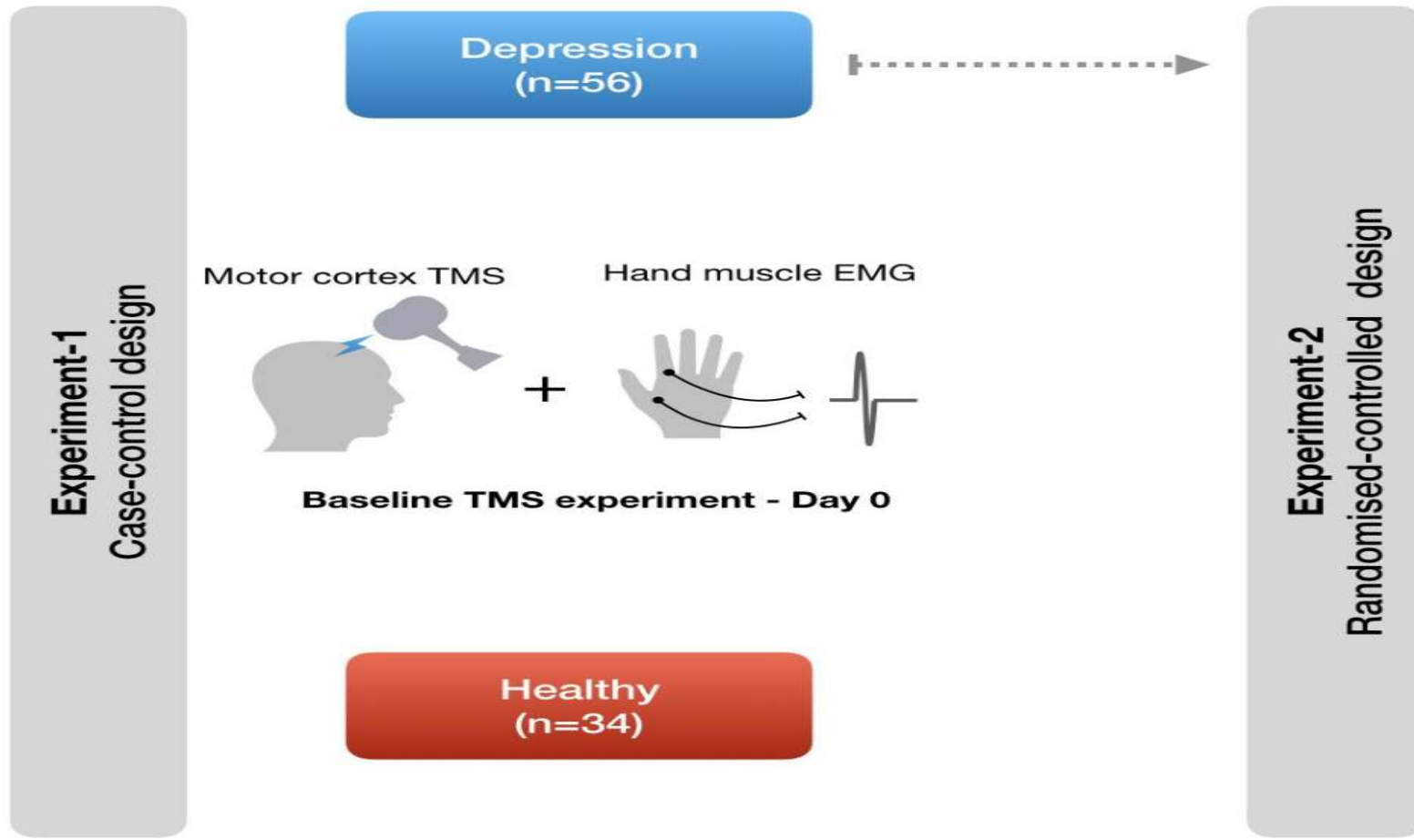

